\title{
Requirements for survivin in terminal differentiation of erythroid cells and maintenance of hematopoietic stem and progenitor cells
}

\author{
Cindy G. Leung, ${ }^{1,2,4}$ Yanfei Xu, ${ }^{1,2}$ Bretton Mularski, ${ }^{1,2}$ Hui Liu, ${ }^{1,2}$ \\ Sandeep Gurbuxani, ${ }^{3}$ and John D. Crispino ${ }^{1,2,4}$ \\ ${ }^{1}$ Committee on Cancer Biology, ${ }^{2}$ Ben May Institute for Cancer Research, and ${ }^{3}$ Department of Pathology, \\ The University of Chicago, Chicago, IL 60637 \\ ${ }^{4}$ Division of Hematology/Oncology, Northwestern University, Chicago, IL 60611
}

Survivin, which is the smallest member of the inhibitor of apoptosis protein (IAP) family, is a chromosomal passenger protein that mediates the spindle assembly checkpoint and cytokinesis, and also functions as an inhibitor of apoptosis. Frequently overexpressed in human cancers and not expressed in most adult tissues, survivin has been proposed as an attractive target for anticancer therapies and, in some cases, has even been touted as a cancer-specific gene. Survivin is, however, expressed in proliferating adult cells, including human hematopoietic stem cells, T-lymphocytes, and erythroid cells throughout their maturation. Therefore, it is unclear how survivin-targeted anticancer therapies would impact steady-state blood development. To address this question, we used a conditional gene-targeting strategy and abolished survivin expression from the hematopoietic compartment of mice. We show that inducible deletion of survivin leads to ablation of the bone marrow, with widespread loss of hematopoietic progenitors and rapid mortality. Surprisingly, heterozygous deletion of survivin causes defects in erythropoiesis in a subset of the animals, with a dramatic reduction in enucleated erythrocytes and the presence of immature megaloblastic erythroblasts. Our studies demonstrate that survivin is essential for steady-state hematopoiesis and survival of the adult, and further, that a high level of survivin expression is critical for proper erythroid differentiation.

CORRESPONDENCE

John Crispino:

j-crispino@northwestern.edu

Abbreviations used: $\mathrm{CBC}$, complete blood count; cdk, cyclin-dependent kinase; CPC, chromosomal passenger complex; E, embryonic day; HE, hematoxylin and eosin; IAP, inhibitor of apoptosis protein; INCENP, inner centromere protein; pI-pC, polyinosinicpolycytidylic acid; RNAi, RNA interference.
At $16.5 \mathrm{kD}$, survivin is the smallest member of the inhibitor of apoptosis protein (IAP) family. Survivin contains a single BIR domain and a C-terminal $\alpha$-helical coiled coil, but no RING finger or other identifiable domain typically found in IAPs (1). Currently, there are three lines of experimental evidence that support a role for survivin in suppression of apoptosis (2). First, overexpression of survivin has been shown to antagonize cell death initiated via the extrinsic or intrinsic apoptotic pathway. Second, transgenic expression of survivin under the keratin 14 (K14) promoter inhibited apoptosis in vivo (3). Conversely, liver cells that were isolated from survivin heterozygous mice exhibited an increase in apoptosis upon treatment with a lower concentration of Fas ligand (4). Third, molecular antagonists of survivin, including antisense, ribozymes, siRNA, and dominant- negative mutants, resulted in caspase-dependent cell death and enhancement of apoptotic stimuli and anticancer activities in vivo (2). Although the role of survivin in conferring resistance to apoptosis is well established, the context in which it does so and the mechanisms by which it accomplishes this remains debatable. It is likely that survivin mediates its antiapoptotic effect through association with other pro- or antiapoptotic molecules (5-8).

Contrary to its role in apoptosis inhibition, survivin's role in mitosis is unequivocal. Survivin inactivation has been shown to cause defects in chromosome alignment, spindle assembly checkpoint, and cytokinesis, with eventual cell death via mitotic catastrophe (9-12). In Caenorhabditis elegans, embryos and oocytes lacking the survivin homologue Bir-1 displayed chromosomal and spindle defects (13). In addition, 
homozygous survivin gene deletion in mice resulted in embryonic lethality at embryonic day (E) 4.5, with cells exhibiting polyploidy and disrupted microtubules (14).

As expected for a mitotic regulator, survivin is generally expressed in a cell cycle-dependent manner, peaking at $\mathrm{G}_{2}-\mathrm{M}$ and rapidly degraded in $G_{1}(15)$. Survivin localizes to the centromeres during prometaphase/metaphase, translocates to the spindle midzone during anaphase/telophase, and eventually localizes to the midbody during cytokinesis (16-18). Consistent with its dynamic localization, numerous studies have shown that survivin is a component of the chromosomal passenger complex (CPC) that functions in the maintenance of an active spindle assembly checkpoint and in cytokinesis. In addition to survivin, the CPC is comprised of inner centromere protein (INCENP) (19), Aurora-B kinase (20), and Borealin/ Dasra B $(21,22)$. RNA interference (RNAi) knockdown of survivin or its partner proteins in U2OS and HeLa cells disrupts proper targeting of the CPC to centromeres and the central spindle, suggesting that these proteins are mutually dependent on each other for proper localization (21, 23-26). It has been proposed that survivin's specific role within the CPC is to target Aurora B kinase to different components of the mitotic apparatus, where it can phosphorylate its substrates.

A growing number of studies indicate survivin is expressed in normal adult cells, particularly human $\mathrm{CD} 34^{+}$hematopoietic stem and progenitor cells (27-29), T lymphocytes (30, 31), polymorphonuclear neutrophils (32), and vascular endothelial cells $(33,34)$, where it may regulate their proliferation or survival. Most recently, our laboratory has reported that survivin is differentially required for the development of two closely related blood cells, erythroid cells and megakaryocytes (35). During terminal differentiation, erythroid cells continue to express survivin mRNA and protein throughout their maturation, even after cell cycle exit, whereas megakaryocytes express an approximately fourfold lower level of survivin mRNA and no detectable protein $(35,36)$. Functionally, a reduction in survivin expression was found to interfere with erythroid cell formation, whereas its overexpression antagonized growth, maturation, and polyploidization of megakaryocytes (35). Furthermore, survivin-deficient hematopoietic progenitors failed to give rise to either erythroid or megakaryocytic colonies in vitro (35). Based on these studies, survivin appears to have critical roles in normal hematopoietic cells, and the physiological impact of survivin-targeted anticancer strategies on the hematopoietic compartment is unclear.

To elucidate precisely the role of survivin in in vivo hematopoiesis, we induced homozygous and heterozygous deletion of the survivin gene in the hematopoietic compartment in mice, using the $M x 1-C r e$ transgene. We show that complete loss of survivin in homozygous-floxed Mx1-Cre animals resulted in rapid lethality. These mutant animals exhibited markedly hypocellular BM and spleen, a plethora of peripheral blood defects, and striking reductions in multiple hematopoietic progenitors, including hematopoietic stem cells. Surprisingly, acute heterozygous deletion of survivin led to defects in erythropoiesis in a subset of animals. Analysis of the erythroid lineage from spleens of these affected mice displayed a dramatic reduction in enucleated erythrocytes, and the presence of immature erythroblasts that were megaloblastic in nature. Collectively, we show that survivin is crucial for steady-state hematopoiesis in the adult, and that erythroid cells are sensitive to survivin levels.

\section{RESULTS}

\section{Survivin is essential for adult hematopoiesis}

Because survivin deficiency results in periimplantation lethality (14), we used a conditional knockout strategy to study the requirement for survivin in in vivo hematopoiesis. Conditional gene-targeted mice, with all four exons of the survivin gene flanked by loxP sites (31), were bred to mice expressing Cre recombinase under the control of the endogenous $M \times 1$ locus, which can be transiently activated in response to type-I IFN (37). Subsequently, mice harboring the Mx1-Cre transgene and either heterozygous or homozygous floxed alleles of survivin were treated with polyinosinicpolycytidylic acid (pI-pC), which is a synthetic double-stranded RNA, to induce deletion of survivin in the hematopoietic compartment. pI-pC treatment of $M \times 1-C r e / s_{1} r^{f /+}$ and $M \times 1-$ $\mathrm{Cre} / \mathrm{sur}^{f / f l}$ adult mice resulted in robust excision of the survivin floxed allele in BM cells as determined by PCR analysis (Fig. 1 A). To our surprise, administration of pI-pC to $M \times 1-$ Cre/sur ${ }^{f l / f}$ mice caused rapid death (Fig. 1 B). Indeed, all of the animals became moribund within $10 \mathrm{~d}$ of pI-pC treatment. In comparison, the control littermates showed no ill effect after $\mathrm{pI}-\mathrm{pC}$ injection, whereas the heterozygous-deleted mice showed 20\% mortality. Analysis of peripheral blood, BM, and spleen (a prominent site of hematopoiesis in young mice) of survivin-deficient animals revealed a wide spectrum of hematopoietic defects. As clearly evident in Fig. 1 D, there was almost a complete ablation of the BM of Mx1-Cre/surfl/fl mice. Consistent with this gross BM depletion, peripheral blood smears from survivin-deleted animals exhibited marked pancytopenia, including reticulocytopenia (Fig. 1 E). In addition, a plethora of erythrocyte abnormalities, such as red cell fragmentation, anisopoikilocytosis, and hypochromia, were visible. Furthermore, Mx1-Cre/surf/fl mice had smaller and extremely hypocellular spleens (Fig. 1, C and F) caused by a reduced white and red pulp. The red pulp, which is a site of normal extramedullary hematopoiesis in young mice, was paucicellular. Most dramatic was the complete absence of nucleated erythroid progenitors that stained positive for the erythroid-specific marker Ter119 in the spleens of $M \times 1$ $\mathrm{Cre} / \mathrm{sur}^{\mathrm{Al} / \mathrm{fl}}$ mice, as shown by immunohistochemical analysis for Ter119 (Fig. 1 G). Control mice that were treated with pI-pC did not display any of these phenotypes (Fig. 1, $\mathrm{B}-\mathrm{G}$ ). Interestingly, however, we observed an intermediate phenotype in survivin heterozygous-deleted mice (as discussed in Haploinsufficiency of survivin leads to defects in erythropoiesis).

To better understand why the animals die within 2 wk of survivin depletion, we repeated the pI-pC injections on another group of mice ( $8 \mathrm{wk}$ of age) and analyzed their complete 



D
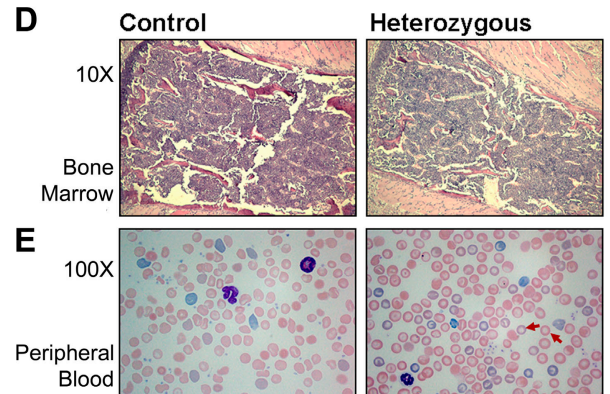

$\mathbf{F}$
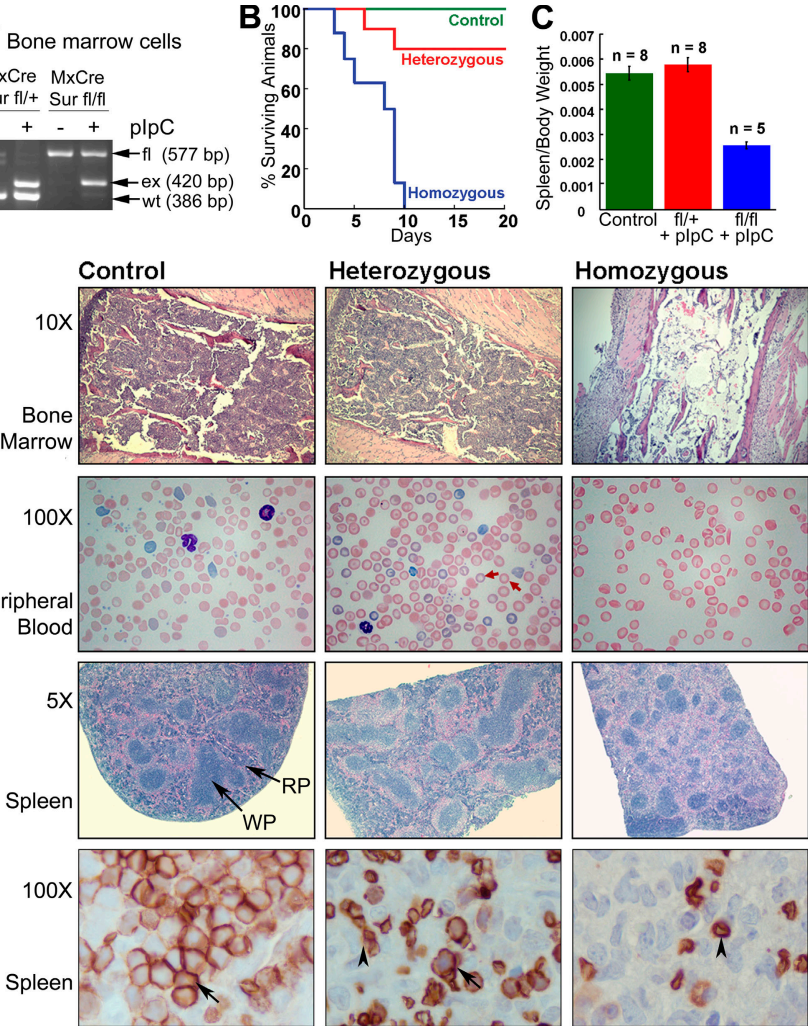

Figure 1. Survivin is essential for adult hematopoiesis. (A) Deletion of the floxed region was monitored by multiplex PCR using three primers to amplify the floxed, excised, and wild-type alleles. PCR of total BM cells from two representative examples $10 \mathrm{~d}$ after $\mathrm{pl}-\mathrm{pC}$ administration. ex, excised; fl, floxed; wt, wild-type. (B) Survival curve for control (Suffl/ ; $n=19$ ), heterozygous (MxCre Surfl/+; $n=10$ ), and homozygous (MxCre Surfl/fl; $n=8$ ) pups after pl-pC treatment. (C) Spleen weight with respect to body weight \pm the SEM was recorded for control, heterozygous, and homozygous-deleted mice. (D) HE stained sections of sternum BM from control, heterozygous, and homozygous-deleted mice. (E) Peripheral blood smears from control, heterozygous, and homozygous-deleted mice were stained with May-Grünwald Giemsa. Red arrows, hypochromic erythrocytes. (F) HE spleen sections from mice in D. WP, white pulp; RP, red pulp. (G) Anti-Ter119 immunohistochemical analysis of spleen sections from mice in D. Black arrows, nucleated Ter $119^{+}$erythroid cells; arrowheads, enucleated Ter $119^{+}$erythrocytes.

blood counts (CBCs) on days 7 and 11 after treatment. CBC analysis at day 7 revealed that survivin-depleted mice had lower hemoglobin and white cell and platelet counts compared with the control animals (unpublished data). By day 11, the survivin-depleted mice developed definitive anemia. Thrombocytopenia and leukopenia were reflected in the automated $\mathrm{CBC}$, as well as on the peripheral blood smears shown in Fig. 1 E; homozygous-deleted animals displayed pancytopenia, with multiple defects in circulating erythrocytes. Therefore, we believe that the development of anemia in the periphery is mostly caused by underproduction, which may or may not be exacerbated by some peripheral destruction. Based on the presence of fragmented red cells in the periphery, we hypothesize that a thrombotic microangiopathic process might contribute to the peripheral destruction of red cells in some of the mice. Furthermore, to investigate if the rapid morbidity and mortality of the survivin-depleted mice was caused by injury to nonhematopoietic tissues, gross and microscopic examination was performed on the major organs from control and survivin-depleted mice. There was no evidence of organ damage in the kidneys, lungs, livers, and hearts of the survivindepleted mice (unpublished data). Under the circumstances, we speculate that the morbidity and mortality observed in the survivin-depleted animals is secondary to BM failure. It is possible that anemia, infections caused by neutropenia, or hemorrhage caused by thrombocytopenia contribute to early morbidity and mortality in these animals.

\section{Hematopoietic progenitors are susceptible to survivin loss}

Because the BM histology indicated that survivin deletion affected multiple hematopoietic lineages, we used flow cytometry to examine specific cell populations in either the BM or spleen of control, Mx1-Cre/sur ${ }^{f /+}$, and Mx1-Cre/sur ${ }^{f / f t}$ mice after $\mathrm{pI}-\mathrm{pC}$ injection. In contrast to littermate controls, $\mathrm{Gr}-1^{+} \mathrm{Mac}-1^{+}$myeloid cells and $\mathrm{CD} 41^{+}$megakaryocytes were both reduced in the $\mathrm{BM}$ of homozygous-deleted mice as early as $36 \mathrm{~h}$ after pI-pC induction (Fig. 2, A and B). C-kit ${ }^{+}$ progenitors were also dramatically diminished in the spleen of homozygous-deleted mice by $10 \mathrm{~d}$ of $\mathrm{pI}-\mathrm{pC}$ administration (Fig. 2 C). Consistent with these results, total BM cells, isolated from homozygous-deleted mice $1 \mathrm{~d}$ after $\mathrm{pI}-\mathrm{pC}$ injection, failed to give rise to any myeloid, erythroid, or megakaryocytic colonies in colony-forming assays (unpublished data). The rapid time frame in which hematopoietic progenitors disappear after survivin excision suggests survivin is critical for the maintenance of hematopoietic cells.

We next focused on analyzing the effect of survivin deletion in the erythroid lineage. During their maturation, erythroid cells progressively become smaller and exhibit chromatin condensation, hemoglobinization, cell cycle exit, and enucleation. As erythroblasts undergo terminal differentiation, they also down-regulate expression of the transferrin receptor CD71 and up-regulate the erythroid-specific marker Ter119. Erythroid cells can be segregated into four subsets by their CD71 and Ter119 expression level (R1 proerythroblasts, Ter119 $9^{\text {low } C D 71 ~ h i g h ~} ;$ R 2 basophilic erythroblasts, Ter119 $9^{\text {high }}$ CD71 ${ }^{\text {high }}$; R3 polychromatophilic erythroblasts, Ter119high CD71 ${ }^{\text {med; }}$ and R4 orthochromatophilic erythroblasts and reticulocytes, Ter119 ${ }^{\text {high }}$ CD71 $71^{\text {low }}$ ) (38). To examine the consequences of survivin deletion specifically on erythropoiesis, we analyzed the distribution of erythroid subsets in the spleen of 1-mo-old $\mathrm{M} \times 1$ - $\mathrm{Cre} / \mathrm{sur}^{f / / A}$ mice $10 \mathrm{~d}$ after $\mathrm{pI}-\mathrm{pC}$ administration. Compared with their littermate controls, homozygousdeleted mice exhibited a marked reduction in the percentages of R1 and R2 subsets and an increase in the percentage of the R4 subset (Fig. 2 D). To investigate the erythroid perturbation more carefully, we isolated R2 and R4 erythroid cells from $\mathrm{pI}-\mathrm{pC}$-treated control and $\mathrm{Mx} 1-\mathrm{Cre} / \mathrm{sur}^{\mathrm{gl} / \mathrm{l}}$ spleens by FACS for morphological evaluation. Because survivin deletion results in rapid BM ablation and early death in $\mathrm{Mx} 1$ - Cre/sur ${ }^{\ell / f l}$ 


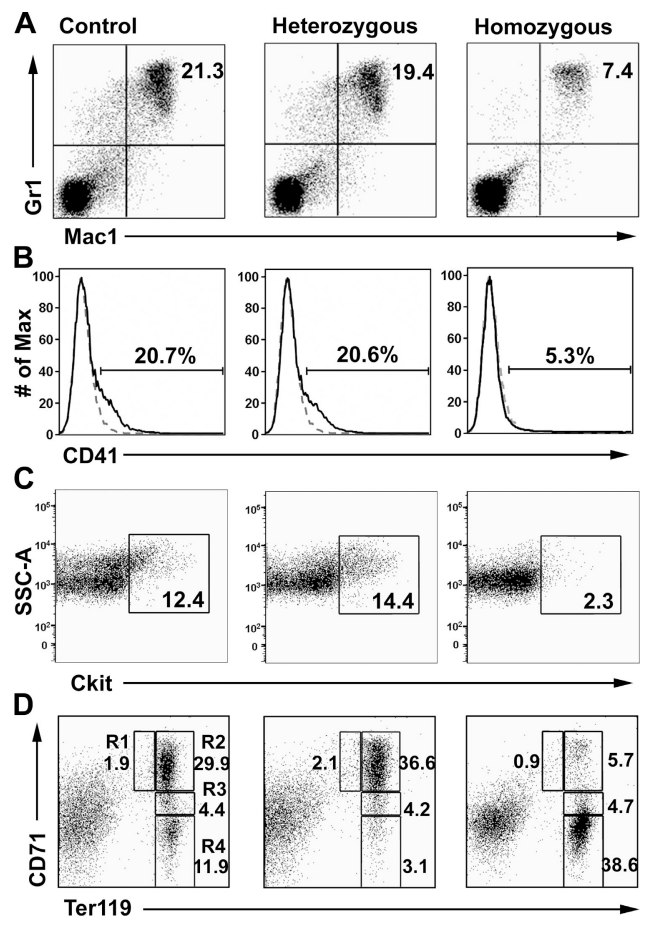

Figure 2. Survivin deletion leads to a reduction in hematopoietic progenitors. (A) $\mathrm{Gr} 1^{+} \mathrm{Mac} 1^{+} \mathrm{BM}$ cells harvested from mice $36 \mathrm{~h}$ after treatment with one dose of $\mathrm{pl}-\mathrm{pC}(n=2)$. (B) $\mathrm{CD} 41^{+} \mathrm{BM}$ cells from mice, as described in $\mathrm{A}$. (C) Flow cytometric analysis of $\mathrm{c}-\mathrm{kit}^{+}$-stained spleen cells from 1-mo-old Sur ${ }^{\mathrm{fl} /+}, \mathrm{MxCre}$ Sur ${ }^{\mathrm{fl}++}$, and MxCre Sur ${ }^{\mathrm{fl} / \mathrm{fl}}$ pups $10 \mathrm{~d}$ after 2 doses of pl-pC. Percentages are shown. (D) CD71+Ter $119^{+}$spleen cells from mice, as described in C. Results shown are representative of four independent experiments.

mice, the experiment was performed at an earlier time point, $4 \mathrm{~d}$ after $\mathrm{pI}$-pC injection. Survivin excision in total spleen cells was confirmed by PCR analysis (Fig. 3 A). At this early time point, the spleens from survivin homozygous-deleted mice were comparable in size to those of control littermates and harbored similar absolute numbers of R 4 cells, but strikingly fewer R2 cells (Fig. 3 B). Morphological evaluation of the R4 population by both May-Grünwald Giemsa and benzidine staining revealed that the majority of cells in survivin-deleted animals were mature, hemoglobinized, and enucleated erythrocytes that were indistinguishable from those of controls (Fig. 3 C). These data support an essential role for survivin in proliferating proerythroblasts and basophilic erythroblasts. In contrast, R 4 cells, which had already exited the cell cycle and extruded their nuclei, were not affected by survivin loss, and the total number of cells within the R4 population was not significantly altered, resulting in an apparent relative increase in the percentage of cells in the R4 gate.

\section{Survivin-depleted erythroblasts exhibit altered cell cycle, increased cell death, and polyploidy}

To determine whether aberrant proliferation or increased cell death was responsible for the erythroid defect, we next analyzed the cell cycle profile and DNA content of survivin-

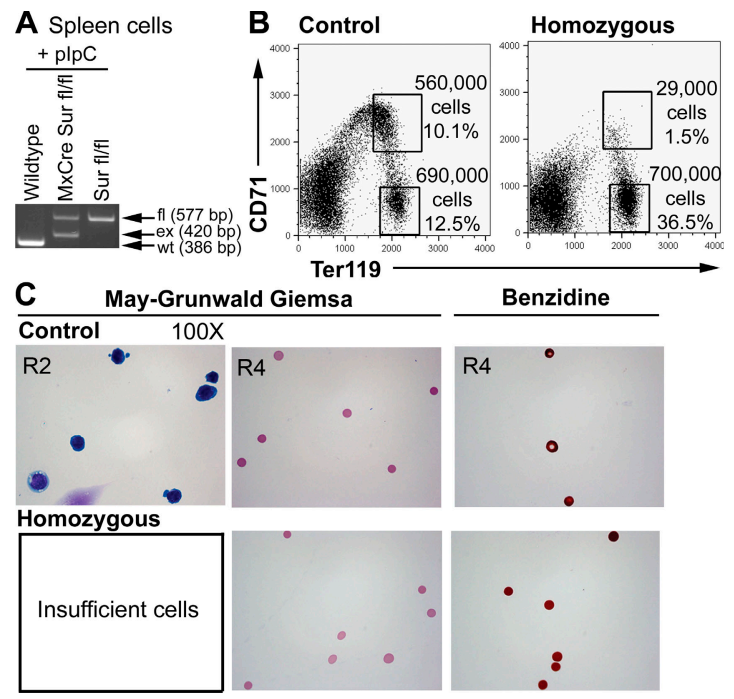

Figure 3. Survivin deletion diminishes proliferating $\mathrm{CD} 71^{+}{ }^{+}$Ter $119^{+}$ erythroid cells. (A) Survivin deletion in total splenic cells from control $\left(\right.$ Sur $\left.{ }^{f / f}\right)$ and homozygous-deleted (MxCre Sur ${ }^{f / f}$ ) mice $4 \mathrm{~d}$ after $\mathrm{pl}-\mathrm{pC}$ treatment was confirmed by PCR. (B) FACS profiles for sorted CD71/Ter119 R2 and R4 erythroid populations are shown with both percentages and absolute cell numbers. (C) Cytospin slides of FACS-sorted R2 and R4 erythroid cells were stained with either May-Grünwald Giemsa or benzidine.

depleted erythroid precursors. BM cells from control and Mx1-Cre/sur ${ }^{f / f l}$ mice were harvested $48 \mathrm{~h}$ after pI-pC treatment and stained with DAPI and antibodies against CD71 and Ter119. Analysis of survivin-deficient erythroblasts showed increased $G_{1}$ and decreased $S$ phase cells, indicating that these cells were either cell cycle-arrested or had exited the cell cycle faster (Fig. 4 A). In addition, survivin-deficient erythroblasts exhibited an increase in cells with $\geq 4 \mathrm{~N}$ DNA content, as well as a moderate increase in cells with sub-G $\mathrm{G}_{1}$ DNA (Fig. 4 B). The increase in the number of cells with sub-G $G_{1}$ DNA suggests that some of the cells have undergone cell death, either as a consequence of aberrant cell cycle progression or initiation of apoptosis.

We next investigated whether the death seen in these cells was a consequence of activation of the intrinsic mitochondrial apoptotic pathway. If survivin depletion resulted in initiation of this pathway, then ectopic Bcl-2 expression in these cells might rescue the phenotype caused by survivin loss. To address this question, we used vav- $B c l-2$ transgenic mice, in which constitutive expression of the human $\mathrm{Bcl}-2$ cDNA is restricted to nucleated hematopoietic cells by the vav promoter (39). To assess the ability of vav-Bcl-2 to promote the survival of erythroid progenitors, lineage-negative $\mathrm{BM}$ cells from vav-Bcl-2 transgenic mice or their wild-type littermates were subjected to varying doses of gamma irradiation $(0,200$, or 300 rads) and plated in erythroid colonyforming assays with IL3, SCF, and EPO. As demonstrated in Fig. $4 \mathrm{C}$, vav- $\mathrm{Bcl}-2$, indeed, promoted the survival of erythroid progenitors in response to gamma irradiation. In fact, after treatment with 200 or 300 rads of gamma irradiation, 

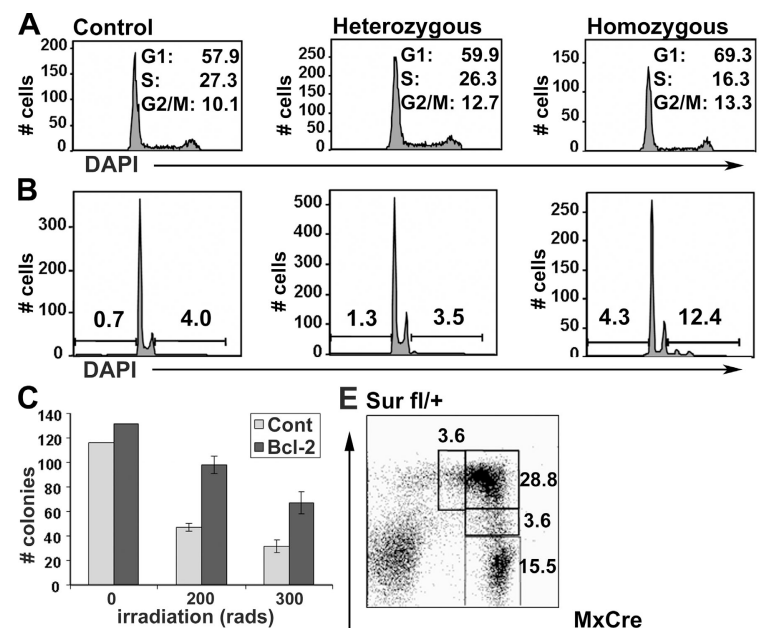

E Sur fl/+
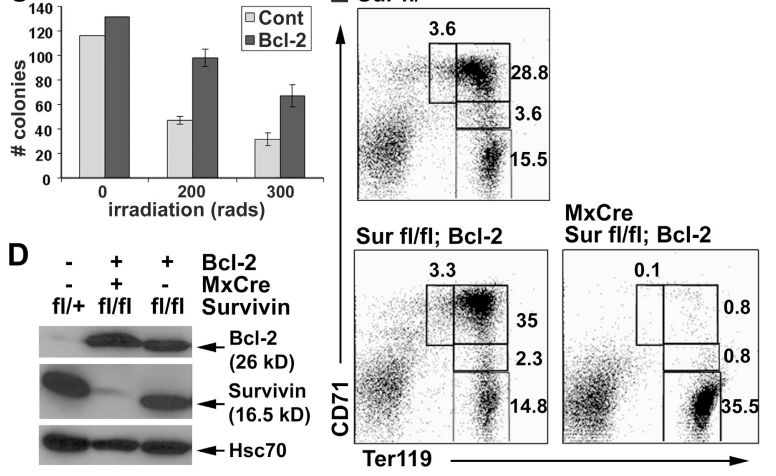

Figure 4. Survivin homozygous-deleted erythroid cells exhibit altered cell cycle, increased cell death, and polyploidy. (A) Control (Surfl/+), heterozygous (MxCre Surfl/+), and homozygous (MxCre Surfl/f) mice were treated with one dose of $\mathrm{pl-pC}$. BM cells were isolated $48 \mathrm{~h}$ later and stained for CD71, Ter119, and DAPI for flow cytometric cell cycle analysis. Cell cycle phases on gated proliferating $\mathrm{CD} 71^{+}$Ter $119^{+}$cells were calculated using Watson Pragmatic model. The data are representative of three sample sets from two independent experiments. (B) SubG1 DNA and ploidy analysis for A. (C) Erythroid colony-forming assays of untreated or gamma-irradiated (200 or 300 rads; $n=3$ ) lineage-negative BM cells from vav-BCl-2 transgenic mice or their wild-type littermates. Mean numbers of pure and mixed erythroid colonies \pm the SEM are shown. (D) Western blot, probed for survivin and Bcl-2, of spleen extracts prepared from Surfl/, Surflff; BCl-2, and MxCre Surfl/fl; BCl-2 pups $10 \mathrm{~d}$ after 2 doses of pl-pC treatment. Heat shock protein 70 (Hsc70) was included as a loading control. (E) Flow cytometric analysis of CD71/Ter119 stained spleen cells from mice, as described in C. Results are representative of five out of seven $\mathrm{MxCre}$ Surflfl; $B \mathrm{BCl}-2$ pups from four independent experiments.

the $v a v-B c l-2$ transgene led to a twofold increase in erythroid colony formation in vitro, compared with wild-type controls. Furthermore, vav- $\mathrm{Bcl}-2$ also prolonged the survival of erythroid progenitors after $3 \mathrm{~d}$ of EPO deprivation (unpublished data). To determine whether overexpression of Bcl-2 can rescue the defects caused by survivin deficiency, we crossed $v a v-B c l-2$ transgenic mice to $M x 1-C r e / s u r^{f / f l}$ mice to generate $\mathrm{Mx1}$-Cre/sur $\mathrm{flf}^{\mathrm{flf}}$; $\mathrm{Bcl}-2$ animals. Bcl-2 and survivin expression were detected by Western blot analysis of protein extracts prepared from the spleens of $\mathrm{pI}-\mathrm{pC}-$ treated control, $\mathrm{Bcl}-2$ transgenic, or $\mathrm{M} x 1$-Cre/sur $\mathrm{r}^{\mathrm{Al} / \mathrm{f}}$; $\mathrm{Bcl}$-2 mice (Fig. 4 D). Although ectopic expression of Bcl-2 under the vav promoter protected erythroid cells on a wild-type survivin background, it failed to rescue the smaller spleen, loss of erythroid progenitors, and eventual demise of $\mathrm{M} \times 1-\mathrm{Cre} / \mathrm{sur}^{\mathrm{fl} / \mathrm{f}}$; $\mathrm{Bcl}-2$ mice after survivin deletion (Fig. 4 E).

\section{Haploinsufficiency of survivin leads to defects in erythropoiesis}

We previously reported that heterozygous loss of survivin caused a nearly $50 \%$ reduction in erythroid colony formation, but a much less dramatic change in the number of megakaryocytic colonies (35). These findings suggested that the erythroid lineage might be uniquely sensitive to the dosage of survivin. In this current study, we observed defects in a proportion of the heterozygous-deleted mice that are consistent with a dose-dependence for survivin in erythroid cells. First, 2 out of the $10 \mathrm{Mx} 1$-Cre/sur fll animals, but none of the control animals, died within $10 \mathrm{~d}$ of $\mathrm{pI}-\mathrm{pC}$ treatment (Fig. $1 \mathrm{~B}$ ). Second, four out of the five BM sections from heterozygousdeleted mice were identified as harboring fewer erythroid precursors in a blinded analysis by a pathologist (S. Gurbuxani; Fig. $1 \mathrm{D}$ and not depicted). Additionally, peripheral blood smears from these mice displayed an increase in hypochromic erythrocytes (Fig. 1 E, red arrows), which were not observed in any $\mathrm{pI}-\mathrm{pC}$-treated control animals.

Furthermore, analysis of the CD71/Ter119 erythroid population by flow cytometry revealed that $20 \%$ of the heterozygous-deleted animals harbored an altered proportion of erythroid cells, with affected animals displaying an increased proportion of $\mathrm{R} 2$ cells and a concomitant decrease in the R4 population (Fig. $2 \mathrm{D}$ and Fig. $5 \mathrm{~A}$ ). In the data depicted in Fig. $5 \mathrm{~A}$, which are representative of the affected group of animals, nearly half as many R 4 cells and a greater number of $\mathrm{R} 2$ cells were isolated from the heterozygous-deleted spleen in comparison to the control spleen. To further explore the nature of this defect, R2 and R4 cells from pI-pC-treated control, and Mx1-Cre/sur fll+ mice were collected by FACS. Excision of the floxed allele was confirmed by PCR analysis (Fig. 5 B). May-Grünwald Giemsa staining of purified cells revealed that although the $\mathrm{R} 2$ progenitors appeared similar, the R4 erythroid population was distinctly different in the two groups (Fig. 5, C and D). Heterozygous survivindeficient R4 cells exhibited a striking increase in the proportion of nucleated erythroblasts, with a concomitant decrease in enucleated erythrocytes. Interestingly, the R4 population of survivin heterozygous-deleted erythroblasts appeared immature and possessed less condensed, larger nuclei, which is indicative of a block in terminal differentiation. Although this phenotype was not fully penetrant, the megaloblastic features observed in a significant proportion of heterozygous-deleted animals suggest that survivin plays a novel role in erythroid terminal differentiation.

\section{Defects caused by survivin deletion are cell autonomous}

Whereas the preceding data suggest that survivin deficiency results in impaired proliferation and subsequent loss of hematopoietic progenitors, Mx1-Cre excision is not absolutely limited to hematopoietic cells (37). Therefore, to determine whether the requirement for survivin is cell autonomous, we transplanted total CD $45.2^{+}$-nucleated BM cells from control,

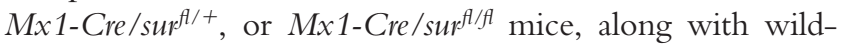
type CD $45.1^{+} / \mathrm{CD} 45.2^{+}$support cells, into lethally irradiated 


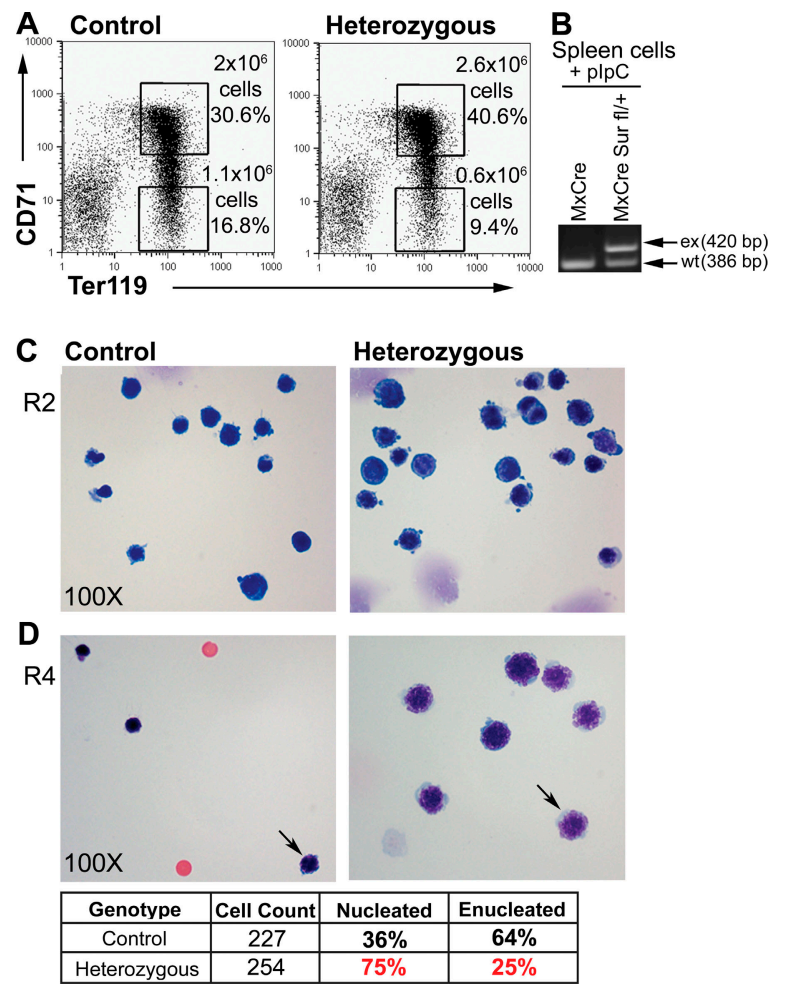

Figure 5. Survivin haploinsufficiency in erythroid maturation. (A) FACS sort profile of CD71/Ter119-stained R2 and R4 erythroid populations from spleens of control ( $\mathrm{MxCre}$ ) and heterozygous-deleted ( $\mathrm{MxCre} \mathrm{Surfl++}$ ) mice $10 \mathrm{~d}$ after $\mathrm{pl}-\mathrm{pC}$ administration. Percentages and absolute cell numbers are shown. (B) Survivin deletion in total splenic cells from $\mathrm{MxCre}$ and MxCre Surfl/+ mice was confirmed by PCR. (C) Cytospin slides of FACS-sorted R2 erythroid cells stained with May-Grünwald Giemsa. (D) May-Grünwald Giemsa-stained cytospin slides of FACS-sorted R4 erythroid cells. Arrows, nucleated erythroblasts. (bottom) The table summarizes percentages of erythroblasts and erythrocytes for the sorted R4 population from each mouse.

CD $45.1^{+}$congenic recipients (Fig. 6 A). Engraftment of donor cells was first verified by staining for CD 45 surface marker expression in peripheral blood $5 \mathrm{wk}$ after transplantation. The engraftment of CD $45.2^{+}$cells ranged from 7 to $69 \%$ (Fig. 6 B). Recipients were then treated with pI-pC, and the contribution of CD $45.2^{+}$cells to the hematopoietic compartment was assessed. Survivin excision led to pronounced depletion of CD $45.2^{+}$cells in the peripheral blood, supporting a role for survivin in hematopoietic stem and progenitor cells (Fig. 6 C). Administration of $\mathrm{pI}-\mathrm{pC}$ to $\mathrm{M} \times 1$-Cre/sur ${ }^{\text {t/fl }}$ transplanted mice also led to near complete disappearance of the BM CD $45.2^{+}$hematopoietic cells within 2 wk of pI-pC treatment in all five mice analyzed (Fig. 6, D and E). No significant changes were observed in either control or Mx1Cre/sur fll ${ }^{f l}$ transplanted mice. These data show that there is a cell autonomous requirement for survivin in hematopoietic progenitors. Next, to determine whether HSCs are also susceptible to survivin deletion, we elucidated the origin of the HSC populations in control and $M x 1-C r e / s u r^{f / f l}$ transplanted
A
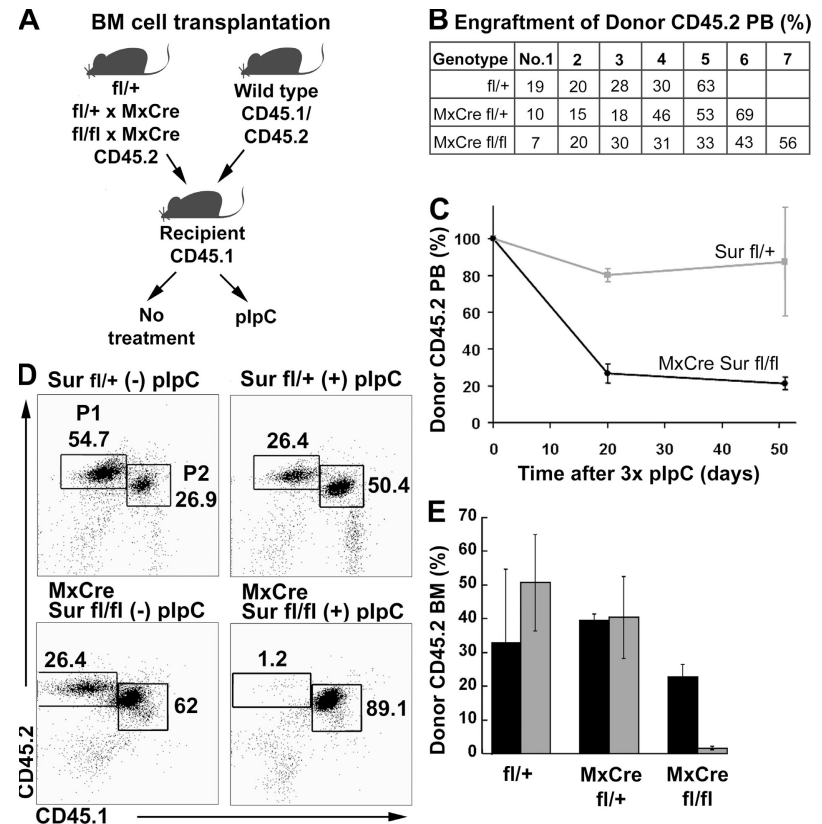

Figure 6. Cell-autonomous defects of survivin deletion. (A) Mice with chimeric BM were generated by transplanting conditionally gene targeted CD45.2+ BM cells with wild-type CD 45.1+/CD45.2+ BM support cells into lethally irradiated congenic $C D 45.1^{+}$recipients. A subset of mice was treated with $\mathrm{pl}-\mathrm{pC}$ after BM transplantation. (B) Table depicts percentage of donor $\mathrm{CD} 45.2^{+}$cells that has engrafted for each mouse before $\mathrm{pl}$-pC administration, as determined by CD45.1/CD45.2 staining of peripheral blood. (C) Mean percentages of CD $45.2^{+}$cells \pm the SEM in the peripheral blood of Surfl/t $(n=3$; gray line) and $M x C r e \operatorname{Surflff}(n=9$; black line) mice after $\mathrm{pl-pC}$ treatment. Initial engraftment of CD45.2 ${ }^{+}$was normalized to $100 \%$ for each mouse. (D) Flow cytometric analysis of CD45.1/ CD45.2 stained BM cells from representative Sur ${ }^{\mathrm{fl} /+}$ and $\mathrm{MxCre}$ Sur ${ }^{\mathrm{fl} / \mathrm{fl}}$ mice in the absence or presence of $\mathrm{pl}-\mathrm{pC}$ treatment. Percentages of $\mathrm{CD} 45.2^{+}$ cells (P1) and CD45. $1^{+} /$CD $45.2^{+}$cells (P2) were determined for gated large nucleated cells. (E) Histogram depicts mean contribution of BM CD45.2 ${ }^{+}$ cells \pm the SEM of Surfl/t $(n=2$, untreated; $n=3$, plus pl-pC), MxCre Surflit+ $(n=2$, untreated; $n=3$, plus pl-pC), and MxCre Surfl/fl $(n=2$, untreated; $n=5$, plus $\mathrm{pl}-\mathrm{pC}$ ) transplanted mice.

animals 10 wk after pI-pC administration. Flow cytometric analyses revealed that the $\operatorname{lin}^{-} \mathrm{c}-\mathrm{kit}^{+} \mathrm{Sca}-1^{+} \mathrm{HSC}$ populations in $\mathrm{Mx} 1-\mathrm{Cre} / \mathrm{sur}^{\mathrm{Al} / \mathrm{fl}}$ transplanted mice were exclusively derived from wild-type CD 45.1+/CD 45.2 $2^{+}$support cells, whereas the HSC populations in control animals expressed either CD45.2 $2^{+}$ alone or CD $45.1^{+/ C D} 45.2^{+}$cell surface markers (Fig. 7).

\section{DISCUSSION}

We show that survivin is an essential gene in the adult and is indispensable for the proliferation and maintenance of both hematopoietic stem and progenitor cells. Similar to inducible deletion of the antiapoptotic Bcl-2 family member myeloid leukemia-1 (MCL-1) in mice, homozygous deletion of survivin resulted in rapid death, ablation of the BM, and loss of early BM progenitor populations, including HSCs (40). Consistent with our findings, $\mathrm{T}$ lineage-specific survivin knockout mice revealed critical requirements for survivin in the 
development and homeostasis of $\mathrm{T}$ cells $(30,31)$. In response to mitogen-induced proliferation, survivin-deficient $\mathrm{T}$ cells exhibited p53-mediated cell cycle arrest, a spindle formation defect, and spontaneous cell death. In addition, p53 inactivation or introduction of $\mathrm{Bcl}-2$ failed to rescue the thymocyte developmental defects caused by survivin deficiency (30). In light of these published works, our study suggests survivin may function as both a chromosomal passenger protein and an inhibitor of apoptosis in the maintenance of hematopoietic stem and progenitor populations.

In addition, we demonstrate that haploinsufficiency of survivin affects erythroid terminal maturation. Erythroid progenitors undergo a series of three to four rapid "differentiation divisions" to give rise to mature, enucleated, and hemoglobinized erythrocytes (41). During this process, the G1 phase of the cell cycle is reduced from $11 \mathrm{~h}$ to $5 \mathrm{~h}$, and the cell size is reduced from a $12-\mu \mathrm{m}$ proerythroblast to a $4-\mu \mathrm{m}$ erythrocyte (mouse). This altered cell cycle progression is accompanied by extensive changes in gene expression, including a rapid down-regulation of D-type cyclins and their partner, cyclindependent kinase type $4(\mathrm{cdk} 4)$. In contrast, expression levels of $\mathrm{S}-$ and $\mathrm{G}_{2}-\mathrm{M}$-associated cell cycle regulators, such as cyclin A and $\mathrm{cdk} 1 / \mathrm{cdc} 2$, are transiently up-regulated. In this study, we show a dose-dependent requirement for survivin in erythropoiesis. We previously reported that survivin levels increase as erythroid cells mature, with both mRNA and protein present in cells throughout even the late orthochromatic stage. In fact, erythroid cells express approximately fourfold higher levels of survivin mRNA and protein than the related megakaryocytic lineage during their terminal differentiation (35). Therefore,

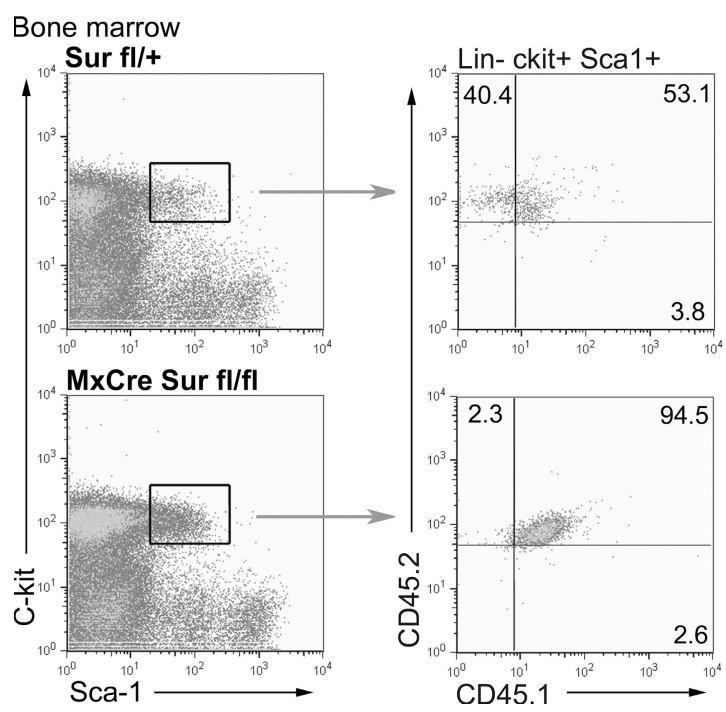

Figure 7. Hematopoietic stem cells are susceptible to survivin deletion. The c-kit and Sca-1 profiles of lineage-negative BM cells (negative for CD5, B220, CD11b, Ter119, Gr-1, 7-4) from Sur ${ }^{f /+}(n=1)$ and $M x C r e S u r f / f f(n=2)$ transplanted mice 10 wk after 3 doses of $\mathrm{pl}-\mathrm{pC}$ (left plots). Lin- ${ }^{-}-\mathrm{kit}^{+} \mathrm{Sca}-1^{+}$cells were gated and analyzed for CD45.1 and CD45.2 cell surface markers (right plots). Numbers indicate percentages of cell populations. reduced levels of key mitotic regulators, including survivin, may compromise the efficiency of the cell cycle machinery and subsequently, lead to defects in erythroid maturation. Consistent with this model, mice heterozygous for BubR1, which is a spindle assembly checkpoint protein, exhibited decreased erythropoiesis and a higher frequency of anemia (42). Interestingly, stable association of BubR 1 to kinetochores and maintenance of the spindle assembly checkpoint is critically dependent on survivin $(12,24)$. In the presence of reduced survivin levels, Aurora B kinase, INCENP, and Borealin may less efficiently localize to different components of the mitotic apparatus, including the inner centromeres, where the CPC plays an important auxiliary role in spindle checkpoint surveillance. Because Aurora B, INCENP, and survivin form a complex that is required for multiple aspects of mitosis and cytokinesis, a reduction in survivin levels likely disrupts proper targeting of Aurora B kinase to its substrates. It remains to be determined whether survivin has a novel role in erythroid terminal differentiation independent of its mitotic function.

Several preclinical studies have shown that disrupting survivin expression or function in cancer cells decreases their proliferation and enhances apoptosis (43). The absence of significant side effects on hematopoietic cells reported in these studies may be caused by local intratumor injection and minimal systemic dissemination of anti-survivin therapies. More recently, shepherdin, which is a small peptide that destabilizes survivin by blocking its interaction with the molecular chaperone heat shock protein 90 (Hsp90), has been shown to affect human $\mathrm{CD} 34^{+}$hematopoietic progenitor colony formation in vitro (44). In that study, treatment of $\mathrm{CD} 4^{+}$cells with shepherdin inhibited precursor erythroid burst-forming unit colony formation and, at slightly higher peptide concentrations, granulocyte-macrophage colony-forming units and granulocyte erythrocyte macrophage megakaryocyte colony-forming units. Our study confirms that the hematopoietic system is extremely sensitive to survivin disruption and emphasizes the need for innovative and selective anti-survivin cancer therapies.

\section{MATERIALS AND METHODS}

Generation of $\mathrm{Mx1}-\mathrm{Cre} / \mathrm{Su} \boldsymbol{r}^{f / f l}$ and $\mathrm{Mx1-Cre} / \mathrm{Su} \boldsymbol{r}^{f / f l}$; Bcl-2 mice. Conditionally gene-targeted mice, with all four exons of the survivin gene flanked by loxP sites, were obtained from A. Winoto (University of California, Berkeley, Berkeley, CA) (31). Mx1-Cre transgenic mice harboring Cre recombinase under the control of the interferon-responsive $M x 1$ promoter in a C57BL/6 background were purchased from The Jackson Laboratory. Vav$\mathrm{Bcl}$-2 transgenic mice, in which constitutive expression of a human $\mathrm{Bcl}-2$ cDNA is driven by the promoter of the vav gene, were provided by J. Adams (Walter and Eliza Hall Insitute of Medical Research, Victoria, Australia) and K. Macleod (University of Chicago, Chicago, IL) (39). To generate Mx1Cre/sur $r^{f l+}$ mice, sur ${ }^{f / f l}$ mice were crossed to $M x 1$-Cre transgenic mice. Mx1$\mathrm{Cre} / \operatorname{sur}^{f /+}$ mice were subsequently bred to $\operatorname{sur}^{f / / f}$ mice to produce littermates with the following genotypes: $\operatorname{sur}^{f l+}$, sur ${ }^{f / f l}, M x 1-C r e / s u r^{f l+}$, and Mx1-Cre/ sur $^{f / f}$. To generate $M x 1-C r e / s u r^{f l / f} ; B c l-2$ mice, $M x 1-C r e / s u r^{f / f l}$ mice were crossed to $\mathrm{Bcl}-2$ transgenic mice to obtain $\mathrm{Mx} 1-\mathrm{Cre} / \mathrm{sur}^{\mathrm{fl} /+}$; $\mathrm{Bcl}-2$ mice, which were then bred to $\operatorname{sur}^{\mathrm{fl}++}$ mice. All animal research was approved by the University of Chicago and Northwestern University Institutional Animal Care and Use Committees. 
Mouse genotyping. Mouse genotyping was performed by PCR of tail DNA. For genotyping the Mx1-Cre transgene, the Mx1-Cre forward $\left(5^{\prime}-\right.$ gcctgcattaccggtcgatgcaacga- $\left.3^{\prime}\right)$ and $M x 1$-Cre reverse $\left(5^{\prime}\right.$-gtggcagatggcgcggcaacaccatt- $3^{\prime}$ ) primers were used to amplify a $725-\mathrm{bp}$ PCR product. Three primers (Adv17, Adv25, and Adv28) were used to genotype the wildtype, floxed, and Cre-deleted survivin allele, as previously described (31). For genotyping the $v a v-B c l-2$ transgene, primers against the SV40 poly(A) DNA sequence were used (39).

Histological analysis. Newborn (10-14 d) mice received two intraperitoneal injections (13 $\mu \mathrm{g} / \mathrm{g}$ body weight) of pI-pC (GE Healthcare) every other day to induce survivin deletion. Sternum BM and spleen were isolated $10 \mathrm{~d}$ later, fixed overnight in formalin (Sigma-Aldrich), and processed for histological analysis. Serial sections were stained with hematoxylin and eosin (HE), and peripheral blood smears were stained with May-Grünwald Giemsa using standard protocols. Immunohistochemistry was performed on deparaffinized spleen sections with anti-Ter119 antibody (BD Biosciences).

Flow cytometric analysis and cell sorting. To obtain BM cells, mouse femurs and tibiae were flushed with PBS, and cells were separated by passage through a 20-gauge syringe. For spleen cells, spleens were cut into small pieces and subsequently disaggregated with an 18-gauge syringe. Cells were resuspended in PBS containing $1 \mathrm{mM}$ EDTA and 1\% bovine serum albumin (Sigma-Aldrich) to reduce aggregates and nonspecific staining, respectively. The cells were stained with different combinations of c-kit, Gr-1, Mac-1, CD41, CD71, and Ter119 antibodies, conjugated to APC, PE, or FITC (BD Biosciences). Finally, surface marker expression was analyzed using a FACSCanto flow cytometer (BD Biosciences) and FlowJo software. To isolate discrete erythroid populations, spleen cells were first stained with FITC-conjugated CD71 and PE-conjugated Ter119 antibodies. Then, based on the CD71 and Ter119 expression level, erythroid subpopulations were purified using a MoFlo-HTS cell sorter (DakoCytomation). FACS-sorted cells were cytospun onto slides and stained with either May-Grünwald Giemsa or benzidine Wright Giemsa for morphological evaluation.

Cell cycle analysis. Adult (6-8 wk) mice were injected intraperitoneally with one dose of $\mathrm{pI}-\mathrm{pC}$. BM cells were isolated $48 \mathrm{~h}$ later and stained with FITC anti-CD71 and PE anti-Ter119 antibodies. Cells were fixed with $2 \%$ paraformaldehyde (Sigma-Aldrich) and 70\% ethanol to preserve cell surface antibody-conjugated fluorochrome staining. Subsequently, cells were treated with $2 \mathrm{~N} \mathrm{HCl} / 0.5 \%$ Triton X-100, followed by $0.1 \mathrm{M}$ sodium tetraborate, and DNA was stained with DAPI (Sigma-Aldrich) at $4^{\circ} \mathrm{C}$ overnight. The cell cycle profile of $\mathrm{CD} 71^{+}$Ter119+ ${ }^{+}$erythroid cells was analyzed using a LSR-II flow cytometer (BD Biosciences) and FlowJo software.

Erythroid colony-forming assays. Primary mouse BM cells were collected from $v a v-B c l-2$ transgenic mice and their wild-type littermates and enriched for progenitors with the EasySep negative-selection mouse hematopoietic progenitor-enrichment kit (StemCell Technologies). The cells were either left untreated or irradiated with 200 or 300 rads in a Cesium-137 irradiator. To evaluate erythroid colony formation, 25,000 cells were plated in MethoCult 3234 (StemCell Technologies), supplemented with IL3, SCF, and $10 \mathrm{U} / \mathrm{ml} \mathrm{EPO}$. Pure burst-forming unit erythroid and mixed colonies were enumerated after $10 \mathrm{~d}$.

BM transplantation. C57BL/6 CD45.1 $1^{+}$congenic recipients (6-8 wk) were purchased from The Jackson Laboratory and placed on Bactrimcontaining water $1 \mathrm{wk}$ before lethal irradiation. Mice were irradiated with a total of 1,200 rads in split doses (800 rads, and then 400 rads $4 \mathrm{~h}$ later). Total nucleated BM cells $\left(10^{6}\right)$, marked by the CD $45.2^{+}$allele from untreated control, Mx1-Cre/sur $r^{f /+}$, or Mx1-Cre/sur ${ }^{A / A l}$ mice, along with CD45.1 $1^{+/}$ CD $45.2^{+}$support cells $\left(0.5 \times 10^{6}\right)$, were injected retroorbitally into lethally irradiated CD $45.1^{+}$recipients that were anesthetized with Nembutal (Abbott Laboratories). CD $45.2^{+}$and CD $45.1^{+} / \mathrm{CD} 45.2^{+} \mathrm{BM}$ cells were allowed to engraft for $5 \mathrm{wk}$ after transplantation. After the engrafting, peripheral blood from recipient mice was obtained by tail vein bleeding, subjected to red blood cell lysis, and stained with PE-Cy5.5-conjugated CD45.1 (eBioscience) and FITC-conjugated CD45.2 (BD Biosciences) antibodies to verify donor cell engraftment by flow cytometric analysis. A subset of the recipients was injected with 2 doses of $\mathrm{pI}-\mathrm{pC}$, whereas the remainder served as untreated controls. $\mathrm{BM}$ cells from $\mathrm{pI}-\mathrm{pC}-$ treated and control animals were isolated $2 \mathrm{wk}$ after $\mathrm{pI}-\mathrm{pC}$ administration and analyzed for the presence of CD $45.2^{+}$and CD $45.1^{+} / \mathrm{CD} 45.2^{+}$donor cells. For HSC analysis, BM cells from transplanted mice, $10 \mathrm{wk}$ after 3 doses of $\mathrm{pI}-\mathrm{pC}$, were depleted of mature hematopoietic lineages, expressing surface antigens CD5, CD11b, B220, Gr-1, Ter119, and 7-4, with EasySep (StemCell Technologies). Lineage-negative cells were stained for APC anti-c-kit, PE-Cy7 anti-Sca-1 (eBioscience), FITC anti-CD45.2, and PE-Cy5.5 anti-CD45.1 antibodies and analyzed by FACSCanto flow cytometer.

The authors thank Dr. Astar Winoto for the survivin-floxed mice, Drs. Jerry Adams and Kay Macleod for the vav-bcl2 transgenic mice, Dr. Hanno Hock for advice on Mx1-Cre targeting, The University of Chicago Cancer Research Center, and the Flow Cytometry Core Facility for technical expertise. Additional thanks to Mitchell Weiss, lannis Aifantis, Jonathan Licht, and Hanno Hock for critical review of the manuscript.

This research was supported, in part, by the Elsa U. Pardee Foundation and by National Institutes of Health grants CA101774 and DK074693. J.D. Crispino is a Scholar of the Leukemia and Lymphoma Society.

The authors have no conflicting financial interests.

\section{Submitted: 14 November 2006}

Accepted: 24 May 2007

\section{REFERENCES}

1. Ambrosini, G., C. Adida, and D.C. Altieri. 1997. A novel antiapoptosis gene, survivin, expressed in cancer and lymphoma. Nat. Med. 3:917-921

2. Altieri, D.C. 2003. Survivin, versatile modulation of cell division and apoptosis in cancer. Oncogene. 22:8581-8589.

3. Grossman, D., P.J. Kim, O.P. Blanc-Brude, D.E. Brash, S. Tognin, P.C. Marchisio, and D.C. Altieri. 2001. Transgenic expression of survivin in keratinocytes counteracts UVB-induced apoptosis and cooperates with loss of p53. J. Clin. Invest. 108:991-999.

4. Conway, E.M., S. Pollefeyt, M. Steiner-Mosonyi, W. Luo, A. Devriese, F. Lupu, F. Bono, N. Leducq, F. Dol, P. Schaeffer, et al 2002. Deficiency of survivin in transgenic mice exacerbates Fas-induced apoptosis via mitochondrial pathways. Gastroenterology. 123:619-631.

5. Song, Z., X. Yao, and M. Wu. 2003. Direct interaction between survivin and Smac/DIABLO is essential for the anti-apoptotic activity of survivin during taxol-induced apoptosis. J. Biol. Chem. 278: 23130-23140.

6. Marusawa, H., S. Matsuzawa, K. Welsh, H. Zou, R. Armstrong, I. Tamm, and J.C. Reed. 2003. HBXIP functions as a cofactor of survivin in apoptosis suppression. EMBO J. 22:2729-2740.

7. Dohi, T., K. Okada, F. Xia, C.E. Wilford, T. Samuel, K. Welsh, H. Marusawa, H. Zou, R. Armstrong, S. Matsuzawa, et al. 2004. An IAPIAP complex inhibits apoptosis. J. Biol. Chem. 279:34087-34090.

8. Sun, C., D. Nettesheim, Z. Liu, and E.T. Olejniczak. 2005. Solution structure of human survivin and its binding interface with Smac/Diablo. Biochemistry. 44:11-17.

9. Li, F., E.J. Ackermann, C.F. Bennett, A.L. Rothermel, J. Plescia, S. Tognin, A. Villa, P.C. Marchisio, and D.C. Altieri. 1999. Pleiotropic cell-division defects and apoptosis induced by interference with survivin function. Nat. Cell Biol. 1:461-466.

10. Kallio, M.J., M. Nieminen, and J.E. Eriksson. 2001. Human inhibitor of apoptosis protein (IAP) survivin participates in regulation of chromosome segregation and mitotic exit. FASEB J. 15:2721-2723.

11. Giodini, A., M.J. Kallio, N.R. Wall, G.J. Gorbsky, S. Tognin, P.C. Marchisio, M. Symons, and D.C. Altieri. 2002. Regulation of microtubule stability and mitotic progression by survivin. Cancer Res. 62:2462-2467. 
12. Lens, S.M., R.M. Wolthuis, R. Klompmaker, J. Kauw, R. Agami, T. Brummelkamp, G. Kops, and R.H. Medema. 2003. Survivin is required for a sustained spindle checkpoint arrest in response to lack of tension. EMBO J. 22:2934-2947.

13. Fraser, A.G., C. James, G.I. Evan, and M.O. Hengartner. 1999. Caenorhabditis elegans inhibitor of apoptosis protein (IAP) homologue BIR-1 plays a conserved role in cytokinesis. Curr. Biol. 9:292-301.

14. Uren, A.G., L. Wong, M. Pakusch, K.J. Fowler, F.J. Burrows, D.L. Vaux, and K.H. Choo. 2000. Survivin and the inner centromere protein INCENP show similar cell-cycle localization and gene knockout phenotype. Curr. Biol. 10:1319-1328.

15. Li, F., G. Ambrosini, E.Y. Chu, J. Plescia, S. Tognin, P.C. Marchisio, and D.C. Altieri. 1998. Control of apoptosis and mitotic spindle checkpoint by survivin. Nature. 396:580-584

16. Skoufias, D.A., C. Mollinari, F.B. Lacroix, and R.L. Margolis. 2000 Human survivin is a kinetochore-associated passenger protein. J. Cell Biol. 151:1575-1582.

17. Adams, R.R., M. Carmena, and W.C. Earnshaw. 2001. Chromosomal passengers and the (aurora) ABCs of mitosis. Trends Cell Biol. 11:49-54.

18. Vagnarelli, P., and W.C. Earnshaw. 2004. Chromosomal passengers: the four-dimensional regulation of mitotic events. Chromosoma. 113: 211-222.

19. Cooke, C.A., M.M.S. Heck, and W.C. Earnshaw. 1987. The inner centromere protein (INCENP) antigens: movement from inner centromere to midbody during mitosis. J. Cell Biol. 105:2053-2067.

20. Bischoff, J.R., L. Anderson, Y. Zhu, K. Mossie, L. Ng, B. Souza, B. Schryver, P. Flanagan, F. Clairvoyant, C. Ginther, et al. 1998. A homologue of Drosophila aurora kinase is oncogenic and amplified in human colorectal cancers. EMBO J. 17:3052-3065.

21. Gassmann, R., A. Carvalho, A.J. Henzing, S. Ruchaud, D.F. Hudson, R. Honda, E.A. Nigg, D.L. Gerloff, and W.C. Earnshaw. 2004 Borealin: a novel chromosomal passenger required for stability of the bipolar mitotic spindle. J. Cell Biol. 166:179-191.

22. Sampath, S.C., R. Ohi, O. Leismann, A. Salic, A. Pozniakovski, and H. Funabiki. 2004. The chromosomal passenger complex is required for chromatin-induced microtubule stabilization and spindle assembly. Cell. 118:187-202.

23. Honda, R., R. Korner, and E.A. Nigg. 2003. Exploring the functional interactions between Aurora B, INCENP, and survivin in mitosis. Mol. Biol. Cell. 14:3325-3341.

24. Carvalho, A., M. Carmena, C. Sambade, W.C. Earnshaw, and S.P. Wheatley. 2003. Survivin is required for stable checkpoint activation in taxol-treated HeLa cells. J. Cell Sci. 116:2987-2998.

25. Ditchfield, C., V.L. Johnson, A. Tighe, R. Ellston, C. Haworth, T. Johnson, A. Mortlock, N. Keen, and S.S. Taylor. 2003. Aurora B couples chromosome alignment with anaphase by targeting BubR1, Mad2, and Cenp-E to kinetochores. J. Cell Biol. 161:267-280.

26. Lens, S.M., and R.H. Medema. 2003. The survivin/Aurora B complex: its role in coordinating tension and attachment. Cell Cycle. 2:507-510.

27. Fukuda, S., and L.M. Pelus. 2001. Regulation of the inhibitor-ofapoptosis family member survivin in normal cord blood and bone marrow CD34(+) cells by hematopoietic growth factors: implication of survivin expression in normal hematopoiesis. Blood. 98:2091-2100.

28. Fukuda, S., and L.M. Pelus. 2002. Elevation of Survivin levels by hematopoietic growth factors occurs in quiescent CD34+ hematopoietic stem and progenitor cells before cell cycle entry. Cell Cycle. 1:322-326.
29. Fukuda, S., C.R. Mantel, and L.M. Pelus. 2004. Survivin regulates hematopoietic progenitor cell proliferation through p21WAF1/Cip1dependent and -independent pathways. Blood. 103:120-127.

30. Okada, H., C. Bakal, A. Shahinian, A. Elia, A. Wakeham, W.K. Suh, G.S. Duncan, M. Ciofani, R. Rottapel, J.C. Zuniga-Pflucker, and T.W. Mak. 2004. Survivin loss in thymocytes triggers p53-mediated growth arrest and p53-independent cell death. J. Exp. Med. 199:399-410.

31. Xing, Z., E.M. Conway, C. Kang, and A. Winoto. 2004. Essential role of survivin, an inhibitor of apoptosis protein, in $\mathrm{T}$ cell development, maturation, and homeostasis. J. Exp. Med. 199:69-80.

32. Altznauer, F., S. Martinelli, S. Yousefi, C. Thurig, I. Schmid, E.M. Conway, M.H. Schoni, P. Vogt, C. Mueller, M.F. Fey, et al. 2004 Inflammation-associated cell cycle-independent block of apoptosis by survivin in terminally differentiated neutrophils. J. Exp. Med. 199: 1343-1354

33. Blanc-Brude, O.P., M. Mesri, N.R. Wall, J. Plescia, T. Dohi, and D.C. Altieri. 2003. Therapeutic targeting of the survivin pathway in cancer: initiation of mitochondrial apoptosis and suppression of tumor-associated angiogenesis. Clin. Cancer Res. 9:2683-2692.

34. Mesri, M., M. Morales-Ruiz, E.J. Ackermann, C.F. Bennett, J.S. Pober, W.C. Sessa, and D.C. Altieri. 2001. Suppression of vascular endothelial growth factor-mediated endothelial cell protection by survivin targeting. Am. J. Pathol. 158:1757-1765.

35. Gurbuxani, S., Y. Xu, G. Keerthivasan, A. Wickrema, and J.D. Crispino. 2005. Differential requirements for survivin in hematopoietic cell development. Proc. Natl. Acad. Sci. USA. 102:11480-11485.

36. Zhang, Y., Y. Nagata, G. Yu, H.G. Nguyen, M.R. Jones, P. Toselli, C.W. Jackson, M. Tatsuka, K. Todokoro, and K. Ravid. 2004. Aberrant quantity and localization of Aurora-B/AIM-1 and survivin during megakaryocyte polyploidization and the consequences of Aurora-B/ AIM-1-deregulated expression. Blood. 103:3717-3726.

37. Kuhn, R., F. Schwenk, M. Aguet, and K. Rajewsky. 1995. Inducible gene targeting in mice. Science. 269:1427-1429.

38. Socolovsky, M., H. Nam, M.D. Fleming, V.H. Haase, C. Brugnara, and H.F. Lodish. 2001. Ineffective erythropoiesis in Stat5a $(-/-) 5 \mathrm{~b}(-/-)$ mice due to decreased survival of early erythroblasts. Blood. 98:3261-3273.

39. Ogilvy, S., D. Metcalf, C.G. Print, M.L. Bath, A.W. Harris, and J.M. Adams. 1999. Constitutive Bcl-2 expression throughout the hematopoietic compartment affects multiple lineages and enhances progenitor cell survival. Proc. Natl. Acad. Sci. USA. 96:14943-14948.

40. Opferman, J.T., H. Iwasaki, C.C. Ong, H. Suh, S. Mizuno, K. Akashi, and S.J. Korsmeyer. 2005. Obligate role of anti-apoptotic MCL-1 in the survival of hematopoietic stem cells. Science. 307:1101-1104.

41. Dolznig, H., P. Bartunek, K. Nasmyth, E.W. Mullner, and H. Beug. 1995. Terminal differentiation of normal chicken erythroid progenitors: shortening of G1 correlates with loss of D-cyclin/cdk4 expression and altered cell size control. Cell Growth Differ. 6:1341-1352.

42. Wang, Q., T. Liu, Y. Fang, S. Xie, X. Huang, R. Mahmood, G. Ramaswamy, K.M. Sakamoto, Z. Darzynkiewicz, M. Xu, and W. Dai. 2004. BUBR1 deficiency results in abnormal megakaryopoiesis. Blood. 103:1278-1285.

43. Fukuda, S., and L.M. Pelus. 2006. Survivin, a cancer target with an emerging role in normal adult tissues. Mol. Cancer Ther. 5:1087-1098.

44. Plescia, J., W. Salz, F. Xia, M. Pennati, N. Zaffaroni, M.G. Daidone, M. Meli, T. Dohi, P. Fortugno, Y. Nefedova, et al. 2005. Rational design of shepherdin, a novel anticancer agent. Cancer Cell. 7:457-468. 